\title{
Editorial
}

\section{Is Monitoring Growing Up?}

\section{Le suivi écologique prend-t-il de la maturité?}

\author{
$\underline{\text { Thomas D. Nudds }}^{1}$ and Marc-André Villard ${ }^{2}$
}

"... all observation must be for or against some view if it is to be of any service."

- Charles Darwin

When it comes to birds and the habitats upon which they depend, there is little question that conservationists continue to face significant challenges. Sometimes it might be easy to imagine that little progress is being made, perhaps even that doing science amounts to so much fiddling while Rome burns. After all, the problems are pretty clear, aren't they? So, once in a while, it is a good idea to stop and smell the roses, as the old adage goes. And what a difference a decade has made to the manner in which monitoring is becoming integral to evaluations of cause and effect in the dynamics of bird populations. Although still too often presented as somehow different from research, "bird monitoring ... should first involve clearly defining, quantitatively, the information required for management, conservation and decision-making, in the context of an adaptive management cycle ..." (Francis et al. 2009). In short, which questions are most in need of answers so that potential harm to bird populations can best be effectively and efficiently mitigated? Quantitative targets are required so that monitoring can occur within a clearly defined context and appropriate actions can be triggered if and as necessary. Put another way: What are the hypotheses that might account for the observed dynamics in bird populations?

Of course, "survey and monitoring form the bedrock for conservation" (British Trust for Ornithology 2009), just as observations and patterns form the bedrock for scientific analysis: They are necessary but not sufficient. From a scientific perspective, when patterns in data are generated in the absence of a priori, competing hypotheses about why they might or might not exist, we entertain a significant risk of weak, or outright incorrect, inference with regard to causes of changes in bird populations. Couple that with the potential for observer expectancy bias (Villard and Nudds 2006), and conservationists risk jumping to conclusions that might be unwarranted, and erode the effectiveness and efficiency of conservation efforts.

A recent example may serve to illustrate. The Committee on the Status of Endangered Wildlife in Canada (2008), on the basis of a pattern of a sustained decline in abundance of the Canada Warbler (Wilsonia canadensis), recommended that the species be assigned "Threatened" status. The Committee treated a number of a posteriori hypotheses about the cause of the pattern; habitat loss and degradation featured prominently, particularly that caused by humans. Less emphasis was placed on an alternate hypothesis, namely, a range-wide decline in spruce budworm (Choristoneura fumiferana) not necessarily linked to human activity. Importantly, one purpose of Canada's endangered species legislation is " ... to prevent wildlife species from being extirpated or becoming extinct, to provide for the recovery of wildlife species that are extirpated, endangered or threatened as a result of human activity ... " [italics added] (Minister of Justice 2002). Sleep et al. (2009) pointed out that the Canada Warbler may be more strongly associated with budworm dynamics than

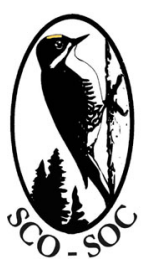

Sponsored by the Society of Canadian Ornithologists and Bird Studies Canada Parrainée par la Société des ornithologistes du Canada et Etudes d'oiseaux Canada

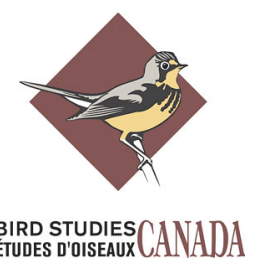


was previously appreciated, and proposed that its decline might be explained by the decline in budworm. Crawford and Jennings (1989) also reported a positive numerical response for this species to a moderate increase in spruce budworm abundance. Venier et al. (2009) concluded that the dynamics of spruce budworm is an important factor when interpreting changes in populations of boreal birds but found no significant association between Canada Warbler and budworm abundances. Whether the available evidence better supports natural, i.e. budworm, or human factors has significant implications for whether the species should be listed at all. If its dynamics are not the result of human activity, what reasonably could humans do to reverse the decline?

Are there systemic reasons why hypotheses about patterns tend often to be treated after the fact, leading potentially to conservation actions that may be less efficient and effective than otherwise? Perhaps. Some formal monitoring protocols emphasize the identification of patterns first and then address the cause. For example, the Integrated Population Approach (IPA) used by Bird Studies Canada (2009) involves "(1) monitoring bird numbers to identify species where the weight-ofevidence points to serious population declines; (2) identifying stages in their life cycles that are associated with these changes; (3) identifying the human or other causes of these changes; (4) proposing remedial or conservation measures; and (5) identifying agencies and other organizations that can take appropriate actions." Similarly, the Integrated Population Monitoring (IPM) Programme (British Trust for Ornithology 2009), after which the IPA was fashioned, aims "(1) to establish thresholds that will be used to notify conservation bodies of requirements for further research or conservation action; (2) to identify the stage of the life cycle at which changes are taking place; (3) to provide data that will assist in identifying the causes of change; and (4) to distinguish changes in populations induced by human activities from those that are natural population fluctuations."

It is fair to ask whether, in future amendments to monitoring protocols, the order implicit in the list of tasks might be revised to better promote the use of stronger, hypothetico-deductive modes of inference over weaker, inductive modes of inference, and reduce the risk that the latter entails for the reliability of the knowledge available to decision makers. This is, in essence, the plea by
Francis et al. (2009). The thorough review of current avian monitoring programs now being undertaken by the Canadian federal government is certainly a chance to try.

“... all observation must be for or against some view if it is to be of any service."

- Charles Darwin

Quand il s'agit des oiseaux et de leur habitat, il n'y a pas de doute que les conservationnistes font face à d'importants défis. Parfois, il peut sembler que les progrès sont modestes, voire même que la science se résume à "bidouiller" pendant que Rome brûle. Après tout, les problèmes sont assez simples, n'estce pas? Donc, c'est parfois une bonne idée de prendre le temps d'arrêter et de sentir les roses, comme le dit le vieil adage. Quelle différence une décennie a pu faire en ce qui a trait à l'intégration du suivi écologique dans l'évaluation des relations de cause à effet dans la dynamique des populations d'oiseaux. Bien que souvent présenté comme étant different de la recherche, le suivi des populations d'oiseaux devrait impliquer d'abord une definition claire et quantitative de l'information requise pour l'aménagement, la conservation et la prise de decisions dans le contexte de l'aménagement adaptatif (Francis et al. 2009). Bref, quelles sont les questions les plus urgentes pour que les impacts potentiels sur les populations d'oiseaux soient contrôlés efficacement et avec diligence? Des cibles quantitatives sont requises pour que le suivi puisse être effectué dans un contexte bien defini et que des actions appropriées puissent être entreprises si nécessaire. En d'autres mots: quelles sont les hypothèses qui pourraient rendre compte de la dynamique observée dans les populations d'oiseaux?

Bien sûr, les inventaires et le suivi forment la pierre angulaire de la conservation (British Trust for Ornithology 2009), tout comme les observations et les patrons sont à la base de l'analyse scientifique: ils sont necessaires, sans être suffisants. D'un point de vue scientifique, quand des patrons dans les données sont observés en l'absence d'hypothèses a priori quant à leur signification, nous courons le risque d'émettre d'inférer de façon peu précise ou carrément erronnée quant aux causes des changements dans les populations d'oiseaux. Ce phénomène, combiné au biais potentiel relié aux 
attentes des observateurs (Villard et Nudds 2006), risque de mener les conservationnistes à tirer des conclusions qui risquent d'être inappropriées et de réduire l'efficacité des efforts de conservation. Récemment, le Comité sur le statut des espèces en péril au Canada (2008) recommandait, sur la base d'un déclin continu de l'abondance de la Paruline du Canada (Wilsonia canadensis), que celle-ci reçoive le statut d'espèce menacée. Le Comité a considéré plusieurs hypotheses a posteriori quant aux causes du patron observé; la perte et la degradation de l'habitat figuraient aux premières loges, en particulier lorsque celles-ci étaient reliées aux activités humaines. Peu d'emphase fut accordée à une hypothèse alternative, soit le déclin à grande échelle de la tordeuse des bourgeons de l'épinette (Choristoneura fumiferana), qui n'est pas nécessairement relié aux activités humaines. Il est important de noter qu'un des objectifs de la législation canadienne en matière d'espèces menacées est d'éviter que les espèces disparaissent, et d'assurer le rétablissement d'espèces disparues du Canada, en voie d'extinction ou menacées en raison de l'activité humaine (Ministre de la justice 2002). Sleep et al. (2009) soutiennent que la Paruline du Canada pourrait être associée plus fortement que l'on pensait à la dynamique de la tordeuse et ils proposent que son déclin pourrait refléter celui de la tordeuse. Crawford et Jennings (1989) ont aussi observé une réponse numérique positive de cette espèce à une augmentation modérée de l'abondance de la tordeuse. Venier et al. (present numéro) concluent que la dynamique des populations de tordeuse est un facteur important à considerer dans l'interprétation des changements dans les populations d'oiseaux boréaux mais ils n'ont observé aucune association significative entre l'abondance de la Paruline du Canada et celle de la tordeuse. Le fait que les données disponibles appuient davantage les facteurs naturels, i.e. la tordeuse, ou les facteurs humains a des implications significatives pour l'inclusion d'une espèce dans la liste des espèces en péril. Si les populations de l'espèce ne reflètent pas les activités humaines, que peuvent faire les humains pour prévenir son déclin?

Y-a-t-il des raisons systémiques pour que les hypothèses sur les patrons de variation soient souvent traitées a posteriori, ce qui mène potentiellement à des interventions de conservation suboptimales? Peut-être. Certains protocoles de suivi mettent l'accent sur l'identification de patrons d'abord, et s'attaquent ensuite aux causes. Par exemple, l'approche intégrée des populations utilisée par Études d'oiseaux Canada (2009) implique (1) le suivi des abondances d'oiseaux afin d'identifier les espèces où l'on observe des déclins importants; (2) l'identification des stades de leur cycle de vie qui sont associés à ces changements; (3) l'identification des facteurs humains ou autres relies à ces changements; (4) la proposition de measures de remédiation ou de conservation; et (5) l'identification d' organisations ou d'institutions qui puissent prendre des actions appropriées. De façon similaire, le programme de suivi intégré des populations du British Trust for Ornithology (2009), qui a inspiré celui d'Études d'oiseaux Canada, vise (1) le développement de seuils qui seront utilisés afin d'alerter des organismes de conservation quant aux besoins en recherche ou en interventions pour la conservation; (2) l'identification des stades du cycle de vie où les changements prennent place; (3) la récolte de données qui permettront d'appuyer l'identification des causes des changements; et (4) la distinction entre les changements dans les populations induits par les activités humaines et ceux qui sont attribuables aux fluctuations naturelles des populations."

Il est approprié de se demander si, lors d'amendements futurs des protocoles de suivi, l'ordre des tâches ne devrait pas être révisé afin de promouvoir l'utilisation de modes d'inférence hypothético-déductifs plus robustes que les approches inductives, afin de réduire le risque que ces dernières comportent pour la qualité des connaisances utilisées dans la prise de décisions. Ceci reflète essentiellement le plaidoyer de Francis et al. (2009). La révision complète des programmes de suivi d'oiseaux récemment entreprise par le gouvernement fédéral du Canada constitue certainement une occasion d'aller dans ce sens.

Responses to this article can be read online at: http://www.ace-eco.org/vol4/iss 1/art7/responses/

\section{LITERATURE CITED}

Bird Studies Canada. 2009. Bird Studies Canada prospectus. Available online at: http://www.bsc-eoc. org/organization/prospectus/prosp11.html.

British Trust for Ornithology. 2009. Integrated population monitoring. Available online at: http:// www.bto.org/survey/ipm.htm. 
Committee on the Status of Endangered Wildlife in Canada (COSEWIC). 2008. COSEWIC assessment and status report on the Canada Warbler Wilsonia canadensis in Canada. COSEWIC, Ottawa, Ontario, Canada. Available online at: http: //dsp-psd.pwgsc.gc.ca/collection 2008/ec/

CW69-14-548-2008E.pdf.

Crawford, H. S., and D. T. Jennings. 1989. Predation by birds on spruce budworm (Choristoneura fumiferana): functional, numerical, and total responses. Ecology 70:152-163.

Minister of Justice. 2002. Species at Risk Act . Available online at: http://laws.justice.gc.ca/PDF/S tatute/S/S-15.3.pdf.

Francis, C. M., P. J. Blancher, and R. D. Phoenix. 2009. Bird monitoring in Ontario: What have we got and what do we need? Forestry Chronicle 85:202-217.

Sleep, D. J. H., M. C. Drever, and K. J. Szuba. 2009. Potential role of spruce budworm in rangewide decline of Canada Warbler. Journal of Wildlife Management 73:546-555.

Venier, L. A., J. L. Pearce, D. R. Fillman, D. K. McNicol, and D. A. Welsh. 2009. Effects of spruce budworm (Choristoneura fumiferana (Clem.)) outbreaks on boreal mixed-wood bird communities. Avian Conservation and Ecology - Écologie et conservation des oiseaux 4(1): 3. [online] URL:

http://www.ace-eco.org/vol4/iss1/art3/.

Villard, M.-A., and T. D. Nudds. 2006. Scientific advocacy: confronting the oxymoron. Avian Conservation and Ecology - Écologie et conservation des oiseaux 1(2): 5. [online] URL: http://www.ace-eco.org/vol1/iss2/art5/. 